\title{
Respiratory Tract Candidiasis in a Tertiary Health Care Unit in Northern India
} Asma Husein Roohani ${ }^{1}$, Nazish Fatima ${ }^{1}$, Haris Manzoor Khan ${ }^{1}$, Anees Akhtar ${ }^{1}$ and Mohammad Shamim ${ }^{2}$

${ }^{1}$ Department of Microbiology, JNMC, AMU, Aligarh, Uttar Pradesh, India

${ }^{2}$ Department of TB and Respiratory Diseases, JNMC, AMU, Aligarh, Uttar Pradesh, India

\begin{abstract}
Respiratory and systemic mycoses are globally emerging as problems of increasing importance in infectious diseases. Among the various opportunistic infections, respiratory infections account for up to $70 \%$ of AIDS defining illnesses $65(43.3 \%)$ samples were positive for fungal elements on culture. Of 65 isolates, $33(50.7 \%)$ were yeasts and $32(49.2 \%)$ were molds. Amongst the yeast isolates, $21(63.6 \%)$ and $12(36.3 \%)$ were collected from immunocompromised and immunocompetent patients respectively. $14(42.4 \%)$ isolates were of $C$. albicans; $64.3 \%$ from the immunocompromised and $35.7 \%$ from the immunocompetent. The remaining $19(57.5 \%)$ isolates of Candida were non albicans candida (NAC). Amongst the $32(49.2 \%)$ mold isolates, $30(93.7 \%)$ were found to be Aspergillus species. A. fumigatus was the most common (53.1\%) followed by $A$. flavus $(31.2 \%)$ and $A$. niger $(9.3 \%)$ Candida isolated from the respiratory secretions does not always indicate invasive candidiasis nor does it indicate the need for antifungal therapy.
\end{abstract}

Keywords: Immunocompromised; Immunocompetent; Non albicans candida (NAC)

\section{Introduction}

Respiratory and systemic mycoses are globally emerging as problems of increasing importance in infectious diseases. Fungal spores are representing more than 50,000 spores per cubic meter of air during the fungal season $[1,2]$. Various mycoses form the bulk of opportunistic infections in AIDS patients and are increasing in the form of an epidemic parallel to the AIDS epidemic [3]. Among the various opportunistic infections, respiratory infections account for up to $70 \%$ of AIDS defining illnesses [4]. Besides the most prevalent and well-known fungal pathogens such as Candida albicans and Aspergillus fumigatus, a large number of new emerging pathogens have been described [57]. Few studies have compared the characteristics between different species in immunocompetent patients [8]. The data on the etiology and spectrum of fungal infections is scarce, particularly in North India. The need of the hour is to undertake more studies on fungal etiological agents especially in a country with a rising population like ours where both the rural and urban masses are potentially at risk. Keeping the above important issues, we undertook this study to find the association of different Candida species in various respiratory tract diseases.

\section{Materials and Methods}

The study was carried out on patients those attending the outpatient department or admitted to ward of Department of TB and respiratory diseases, and the Anti-retroviral clinic at Jawaharlal Nehru Medical College and Hospital, AMU. The period of study was from January 2015 to July 2016.

\section{Selections of cases}

Study group and design: 150 patients were divided amongst 2 subgroups:

i. Immunocompetent: Patients with clinical suspicion of lung carcinoma and chronic lung diseases like interstitial lung disease, chronic obstructive pulmonary disease etc.

ii. Immunocompromised: Patients with weakened immune systems i.e., with significant neutropenia $<500$ neutrophils/ $\mu$ for longer than 10 days. These include AIDS, cancer and transplant patients who are taking corticosteroids, certain immunosuppressive drugs; and those with inherited diseases that affect the immune system (e.g., congenital agammaglobulinemia, congenital IgA deficiency).

A detailed clinical history was recorded for each patient (Proforma attached-Annexures) especially regarding:

i. Onset and duration of illness.

ii. HIV status.

iii. History of smoking/gutka chewing.

iv. Occupation (especially any exposure to grains).

v. Intake of broad-spectrum antibiotics, anti-retroviral treatment, anti -tubercular therapy, corticosteroids, immunosuppressants or any anticancer therapeutics.

vi. Dietary habits.

\section{Collection of specimens}

Expectorated sputum: Early morning samples were collected. The patients were asked to rinse their mouth with normal tap water before collection. Two consecutive samples were considered positive.

Induced sputum: $8 \%$ saline was used to nebulise the patient for 15 minutes for induction of sputum.

Bronchoalveolar lavage: Bronchoalveolar lavage was collected in a clean sterile vial by fibreoptic bronchoscopy after taking written informed consent. The bronchoscope was inserted through the endotracheal tube and wedged in a subsegmental bronchus. Five $20 \mathrm{~mL}$ sterile saline aliquots at room temperature were infused and manually aspirated with a $20 \mathrm{~mL}$ volume syringe. The first aliquot was discarded,

*Corresponding author: Nazish Fatima, Department of Microbiology, JNMC, AMU, Aligarh, Uttar Pradesh, India, Tel: +91-571-2707172; E-mail: nazsham28@gmail.com

Received September 12, 2018; Accepted October 27, 2018; Published November 09, 2018

Citation: Roohani AH, Fatima N, Khan HM, Akhtar A, Shamim M (2018) Respiratory Tract Candidiasis in a Tertiary Health Care Unit in Northern India. J Med Microb Diagn 7: 286. doi:10.4172/2161-0703.1000286

Copyright: @ 2018 Roohani AH, et al. This is an open-access article distributed under the terms of the Creative Commons Attribution License, which permits unrestricted use, distribution, and reproduction in any medium, provided the original author and source are credited. 
and the others were pooled and immediately sent to the laboratory. Pulse oximetry, electrocardiogram, and ventilatory parameters were monitored throughout the procedure.

Endotracheal aspirates: After donning sterile gloves, sampling was performed with a sterile catheter. The catheter was introduced via the endotracheal tube for at least $30 \mathrm{~cm}$, aspirate was suctioned and directly collected into sterile containers. The samples were transported within 15 minutes to the Laboratory of Medical Microbiology.

Pleural fluid: The proposed site for aspiration was directly over a palpable intercostal space and above the level of the diaphragm (no lower than $8^{\text {th }}$ intercostal space). An $18 \mathrm{G}$ cannula was attached to a syringe and the needle was advanced along in the same plane as the local anaesthetic was injected. On collection of $5 \mathrm{ml}$ pleural fluid, the needle was removed leaving the cannula in place which was sealed to prevent entry of air. The catheter was removed with patient at end expiration and the cannula was removed during the breath hold.

Blood: $5 \mathrm{ml}$ blood was collected by venepuncture with all aseptic precautions in sterile plain vial. The vein from which the blood was withdrawn was chosen before the skin was disinfected. If the patient had an existing IV-line, blood was withdrawn below the existing IV line.

\section{Processing of specimens in the microbiological laboratory}

\section{Blood:}

i. Serum was used for HIV testing for the confirmation of HIV status.

ii. Fungal culture in biphasic brain heart infusion Agar/broth.

iii. Serum was used for antigen detecetion test for Cryptococcus by latex agglutination test using 'Cryptococcal antigen latex agglutination test' (Meridian bioscience, Europe).

iv. Serum was used to detect the Aspergillus galactomannan antigen by 'Platelia Aspergillus EIA' (Bio-Rad, Germany).

v. Estimation of CD4 cell counts.

Sputum (Expectorated and induced): Sterile glass beads were added to the sputum sample and vortexed briefly, equal volumes of freshly prepared sodium citrate $(2.94 \%)$ and $0.5 \% \mathrm{~N}$-Acetyl L-Cysteine added to specimen and vortexed again for 10-30 seconds depending upon the consistency, the mixture was diluted in phosphate buffer by adding double the volume and centrifuged at $1000 \mathrm{~g}$ for 15 minutes.

Endotracheal aspirates: Endotracheal aspirate samples were considered valid for culture if $<10$ squamous epithelial cells and $>25$ neutrophils were present.

BAL and pleural fluid: They were directly inoculated for the respective tests. culture:

All of the respiratory samples were subjected following tests before

i. HIV testing by ELISA/RAPID/SIMPLE tests and CD4 cell count estimation: The HIV status of all patients was confirmed at VCTC, Department of Microbiology, JNMC. The HIV antibody assay was assessed by three ERS (ELISA, Rapid, and Simple) tests as recommended by the National AIDS Control Organization (NACO), Ministry of Health and Family Welfare, Government of India (2007). The CD4 cell counts of all the patients were estimated by Flow-Cytometry using Partec CyFlow ${ }^{\circ}$ Counter (Germany) ii. Microscopy: Direct microscopy of the clinical materials was performed by Grams, Giemsa staining, Calcofluor white stain, India ink and $\mathrm{KOH}$ preparation.

iii. Culture for fungus: After initial inoculation and incubation, all culture media were examined for fungal growth daily during the first week and on alternate days thereafter up to 3 weeks. The isolates were identified on macroscopic and microscopic morphological characteristics using standard techniques described in Medical mycology [9].

Identification and characterization of fungal isolates: Yeast isolates were identified on the basis of colony characteristics and further by germ tube production, morphology on corn meal agar $(\mathrm{Hi}$ Media), HiCrome candida agar (Hi Media), urease test, carbohydrate fermentation tests and assimilation tests using yeast nitrogen base agar (Hi Media). Identification and speciation of the molds was done based on the colony characteristics, morphology on lactophenol cotton blue preparation and microslide culture. The suspected molds were further cultured on Czapek Dox agar.

Specialized tests on serum and BAL for antigen detection: Cryptococcal Antigen Latex Agglutination System $\left(\right.$ CALAS $\left.^{\circledast}\right)$ [Meridian Bioscience, Europe] was used to detect Cryptococcal Antigen in Serum and Respiratory samples and The Platelia TM aspergillus EIA (BioRad, Germany) is an immune enzymatic sandwich microplate assay for the detection of Aspergillus galactomannan antigen.

\section{Results}

Most of the patients i.e., 47 (31.3\%) were between 31-40 years with a mean age of 32.5 years. The male to female ratio was $1.8: 1$. Out of 150 patients, the immunocompetent patients comprised of 70 cases whilst the immunocompromised patients comprised of 80 cases. Majority of immunocompetent cases were those presenting with lung mass, i.e., carcinoma (26.6\%) and secondaries in lung (8\%), whilst HIV positive patients constituted the maximum number $(40 \%)$ of immunocompromised cases.

The majority of specimens collected from the patients were induced sputum (40\%) followed by Bronchoalveolar lavage (BAL) (33.3\%). Sputum $(6.6 \%)$, endotracheal aspirate $(6.6 \%)$, pleural fluid $(6.6 \%)$ and Intercostal Tube Drainage (ICTD) (6.6\%) represented the remaining respiratory samples.

$65(43.3 \%)$ samples were positive for fungal elements on culture. Immunocompromised patients showed a higher rate of detection of $26.7 \%$ as compared to $16.7 \%$ in immunocompetent patients which was not statistically significant.

Samples were positive in $35(58.3 \%)$ induced sputum samples, 16 (32\%) BAL samples, 5 (50\%) sputum samples, 2 (20\%) endotracheal aspirates, and $1(10 \%)$ each of pleural fluid and ICTD samples (Tables 1 and 2).

Amongst the 33 (50.7\%) yeast isolates, 21 (63.6\%) and 12 (36.3\%) were collected from immunocompromised and immunocompetent patients respectively. 14 (42.4\%) isolates were of C. albicans; $64.3 \%$ from the immunocompromised and $35.7 \%$ from the immunocompetent. 10 (71.4\%) isolates of these were from patients of pulmonary tuberculosis. The remaining 19 (57.5\%) isolates of Candida were NAC of which, $C$ dubliniensis (12.1\%) and C. glabrata (15.1\%) represented the majority of isolates. $9.1 \%$ were represented each by C. parapsilosis and C. tropicalis. For each of these species, the contribution was either equal or greater in the immunocompromised patients, except for C. tropicalis where $66.6 \%$ 


\begin{tabular}{|c|c|c|c|}
\hline $\begin{array}{l}\text { Clinical } \\
\text { Sample }\end{array}$ & $\begin{array}{c}\text { No. of } \\
\text { immunocompetent } \\
\text { cases positive on culture (\%) } \\
(n=70)\end{array}$ & $\begin{array}{c}\text { No. of } \\
\text { immunocompromised } \\
\text { cases positive on culture (\%) } \\
(n=80)\end{array}$ & $\begin{array}{l}\text { Total } \\
\text { No. of } \\
\text { samples } \\
(n=150)\end{array}$ \\
\hline Sputum & $5(50)$ & 0 & 10 \\
\hline $\begin{array}{l}\text { Induced } \\
\text { sputum }\end{array}$ & 0 & $35(58.3)$ & 60 \\
\hline BAL & $16(32)$ & 0 & 50 \\
\hline Pleural fluid & $1(10)$ & $3(30)$ & 10 \\
\hline ICTD & $1(10)$ & 0 & 10 \\
\hline $\begin{array}{l}\text { Endotracheal } \\
\text { Aspirate }\end{array}$ & $2(20)$ & $2(20)$ & 10 \\
\hline Total & 25 (16.6) & $40(26.6)$ & 150 \\
\hline
\end{tabular}

Table 1: Distribution of fungal isolates with respect to clinical samples.

\begin{tabular}{|c|c|c|c|}
\hline Fungal isolate & $\begin{array}{c}\text { No. of } \\
\text { immunocompetent } \\
\text { cases (\%) } \\
(\mathbf{n = 7 0 )}\end{array}$ & $\begin{array}{c}\text { No. of } \\
\text { immunocompromised } \\
\text { cases (\%) } \\
(\mathbf{n = 8 0 )}\end{array}$ \\
\hline Yeasts & $12(36.3)$ & $21(63.6)$ \\
\hline Molds & $13(40.6)$ & $19(59.3)$ \\
\hline Total & $25(16.7)$ & $43(50.7)$ \\
\hline
\end{tabular}

Table 2: Distribution of fungal isolates with respect to immune status.

isolates were seen in immunocompetent patients as compared to $33.3 \%$ isolate from immunocompromised patients. Cryptococcus neoformans and Pneumocystis jirovecii represented $2(6.1 \%)$ isolates each, all of which were found in HIV positive cases (Table 3 ).

Amongst the 32 (49.2\%) mold isolates, 30 (93.7\%) were found to be Aspergillus species. A. fumigatus was the most common (53.1\%) followed by A. flavus (31.2\%) and A. niger (9.3\%) (Table 4).

\section{Discussion and Conclusion}

Sample positivity was observed in $35(58.3 \%)$ induced sputum samples, 16 (32\%) BAL samples, 5 (50\%) sputum samples, 2 (20\%) endotracheal aspirates, and 1 (10\%) each of pleural fluid and ICTD samples. Singh et al. (2014) demonstrated fungal etiology in respiratory infections from BAL fluid (2.2\%), sputum, endotracheal aspirate, and pleural fluid (1.1\% each).

Amongst the various respiratory samples collected, BAL contributed to $33.3 \%$ of 150 samples and represented majority of the samples from immunocompetent patients. There are series of recommendations for performing BAL fluid, according to European Respiratory Society $[10,11]$. However, there are difficulties in performing BAL fluid collection in some critical patients like hematology patients with complication rates approaching $15 \%$ and patients with borderline oxygenation who require elective pre-procedure intubation and ventilation. Sputum, pleural fluid, intercostal tube drainage, and endotracheal aspirate contributed to $6.6 \%$ each. Samples like the pleural fluid obtained in many cases of fungal pleuritis are a reliable specimen for the diagnosis of pulmonary infections. Induced sputum contributed to $40 \%$ of the total samples $[12,13]$. They represented majority of the samples from HIV positive patients as various studies have shown that chances of isolation of organisms from sputum vary from $30 \%$ to $80 \%$ in HIV positive patients [14].

Of 65 isolates, $50.7 \%$ were yeasts and $49.2 \%$ were molds. Amongst the 33 yeast isolates, $21(63.6 \%)$ and $12(36.3 \%)$ were collected from immunocompromised and immunocompetent patients respectively.

Candida isolates were found in 17 immunocompromised and 12 immunocompetent patients. The isolation of Candida species from the respiratory secretions is frequent in mechanically ventilated patients [15]. Despite the debate about the diagnosis of pulmonary candidiasis, the definite diagnosis of pulmonary candidiasis still rests on histologic demonstration of the yeast in lung tissue with associated inflammation [16]. In 2009, the Infectious Diseases Society of America updated the Clinical Practice Guidelines for the Management of Canadidiasis and stated that Candida isolated from the respiratory secretions does not always indicate invasive candidiasis nor does it indicate the need for antifungal therapy [17]. On the other hand, some researchers believe that, due to the tense situation of the doctor-patient relationship at present, the positive detection of Candida in respiratory secretions without a treatment could contribute to legal actions taken by the patient against the clinician [18].

Of the total 33 isolates, $14(42.4 \%)$ isolates were of C. albicans; $64.3 \%$ from the immunocompromised and $35.7 \%$ from the immunocompetent. 10 (71.4\%) isolates of these were from patients of pulmonary tuberculosis. This is because tuberculosis has always seemed to be associated with many other secondary infections, the commonest among them being Candida spp. infection.

The remaining 19 (57.5\%) isolates of Candida were NAC. Likewise, Non-albicans Candida were isolated from majority (29\%) of patients while C. albicans were isolated from just $26 \%$ of patients in a study of Bharathi et al., [19]. The distribution of NAC in our study was $12.1 \%$ by C. dubliniensis, $15.1 \%$ by C. glabrata, $9.1 \%$ each by C. parapsilosis and C. tropicalis. For each of these species, the contribution was either equal or greater in the immunocompromised patients, except for C. tropicalis where $2(66.6 \%)$ were seen in immunocompetent patients as compared to just $1(33.3 \%)$ isolate from an HIV positive patient. The higher rate of detection can be explained by the longevity of hospitalization of the severely immunocompromised individual (HIV positive patients) that has allowed these species to emerge and cause diseases, of the various NAC, C. dubliniensis is known to be associated with HIV positive patients which supports its higher prevalence in our study [20,21]. C. albicans and C. glabrata were still the most frequently occuring pathogens, there was a decline in the number of $C$. albicans relative to the increase of $C$. glabrata. Trick et al. also observed this phenomenon [22].

Patterns of invasive fungal infections are changing in many ways. In 
Citation: Roohani AH, Fatima N, Khan HM, Akhtar A, Shamim M (2018) Respiratory Tract Candidiasis in a Tertiary Health Care Unit in Northern India. J Med Microb Diagn 7: 286. doi:10.4172/2161-0703.1000286

Page 4 of 4

\begin{tabular}{|c|c|c|c|c|}
\hline \multicolumn{2}{|r|}{ Yeast isolates } & $\begin{array}{l}\text { No. of immunocompetent } \\
\text { cases }(n=12)\end{array}$ & $\begin{array}{l}\text { No. of immunocompromised } \\
\text { cases }(n=21)\end{array}$ & $\begin{array}{c}\text { Total }(\%) \\
(n=33)\end{array}$ \\
\hline \multicolumn{2}{|r|}{ Candida albicans } & $5(35.7)$ & $9(64.3)$ & $14(42.4)$ \\
\hline \multirow{4}{*}{ NAC } & C. dubliniensis & $2(50)$ & $2(50)$ & $4(12.1)$ \\
\hline & C. glabrata & $2(40)$ & $3(60)$ & $5(15.1)$ \\
\hline & C. parapsilosis & $1(33.3)$ & $2(66.6)$ & $3(9.1)$ \\
\hline & C. tropicalis & $2(66.6)$ & $1(33.3)$ & $3(9.1)$ \\
\hline \multicolumn{2}{|r|}{$\begin{array}{l}\text { Cryptococcus } \\
\text { neoformans }\end{array}$} & 0 & $2(100)$ & $2(6.1)$ \\
\hline \multicolumn{2}{|r|}{$\begin{array}{l}\text { Pneumocystis } \\
\text { jirovecii }\end{array}$} & 0 & $2(100)$ & $2(6.1)$ \\
\hline \multicolumn{2}{|r|}{ Total } & $12(36.3)$ & $21(63.6)$ & 33 \\
\hline
\end{tabular}

Table 3: Distribution of yeast isolates in relation to immune status.

\begin{tabular}{|c|c|c|c|}
\hline Mold isolates & No. of immunocompetent cases $(n=13)$ & No. of immunocompromised cases $(n=19)$ & Total $(\%) \quad(n=32)$ \\
\hline A. fumigatus & $8(25)$ & $9(28.1)$ & $17(53.1)$ \\
\hline A. flavus & $4(12.5)$ & $6(18.7)$ & $10(31.2)$ \\
\hline A. niger & 0 & $3(9.3)$ & $3(9.3)$ \\
\hline Mucor & $1(3.1)$ & 0 & $1(3.1)$ \\
\hline $\begin{array}{c}\text { Penicilium } \\
\text { marneffei }\end{array}$ & 0 & $1(3.1)$ & $1(3.1)$ \\
\hline Total & 13 & 19 & 32 \\
\hline
\end{tabular}

Table 4: Distribution of yeast isolates in relation to immune status.

the midst of these evolving trends, IFI of the respiratory tractcontinue to remain important causes of morbidity and mortality. Diagnostic tools can be adequately used only if the treating physician is aware of the propensity of patients to acquire a fungal infection. Thus, continuous awareness and education is crucial for successful management of patients.

\section{References}

1. Pashley CH, Fairs A, Free RC, Wardlaw AJ (2012) DNA analysis of outdoor air reveals a high degree of fungal diversity, temporal variability and genera not seen by spore morphology. Fungal Biol 116: 214-224.

2. Denning DW, Pashley C, Hartl D, Wardlaw A, Godet C, et al. (2014) Fungal allergy in asthma-state of the art and research needs. Clin Transl Allergy 4: 14.

3. Mirdha BR, Banerjee U, Sethi S, Samantry JC, Malviya AN (1993) Spectrum of opportunistic fungal and parasitic infections in hospitalized AIDS patients. CARC Calling 6: 9-10.

4. Walker PA, White DA (1996) Pulmonary disease. Medical Clinics of North America 80: 1337-1362.

5. Horré R, Symoens F, Delhaes L, Bouchara JP (2010) Fungal respiratory infections in cystic fibrosis: A growing problem. Med Mycol 48: 1-3.

6. Fisher MC, Henk DA, Briggs CJ, Brownstein JS, Madoff LC, et al. (2012) Emerging fungal threats to animal, plant and ecosystem health. Nature 484: 186-194.

7. Marguet C, Favennec L, Matray O, Bertout S, Giraud S, et al. (2012) Clinica and microbiological efficacy of micafungin on Geosmithia argillacea infection in a cystic fibrosis patient. Med Mycol Case Rep 1: 79-81.

8. Cao B, Cai BQ, Wang H, Zhou CY, Wang P, et al. (2007) A study of 152 cases of pulmonary fungal infection: Re-evaluation of the microbiological spectra. Zhonghua Jie He He Hu Xi Za Zhi 30: 279-283.

9. Chander J (2009) Medical Mycology. $3^{\text {rd }}$ Edn 24: 556-570

10. Sackner MA, Wanner A, Landa J (1972) Applications of broncho-fiberoscopy. Chest 62: 70-78.
11. Reynolds HY (1991) Immunologic system in the respiratory tract. Physiological Reviews 71: 1117-1133.

12. Murray PV, O'Brien ME, Padhani AR, Powles R, Cunningham D, et al. (2001) Use of first line bronchoalveolar lavage in the immunosuppressed oncology patient. Bone Marrow Transplantation 27: 967-971.

13. Kimura M, Udagawa SI, Makimura K, Satoh K, Toyazaki N, et al. (2009) Isolation and identification of Rhizomucor pusillus from pleural zygomycosis in an immunocompetent patient. Medical Mycology 8: 869-873.

14. Carmichael A, Bateman N, Nayagam M (1991) Examination of induced sputum in the diagnosis of Pneumocystis carinii pneumonia. Cytopathology 292: 61-66.

15. Williams DM, Krick JA, Remington JS (1976) Pulmonary infection in the compromised host. Am Rev Respir Dis 114: 359-394.

16. Haron E, Vartivarian S, Anaissie E, Dekmezian R, Bodey G (1993) Primary Candida pneumonia: Experience at a large cancer center and review of the literature. Medicine 72: 137-142.

17. Pappas P, Rex J, Lee JY, Hamill RJ, Larsen RA, et al. (2003) A prospective observational study of candidemia: epidemiology, therapy, and influences on mortality in hospitalized adult and pediatric patients. Clin Infect Dis 37: 634643.

18. Wen-Ming Xu, Wei Shui, Jian-Cong Lin, Zhen-Qiu Lin, Ming Li, et al. (2015) The serum glucan level and pathological changes of antifungal treatment for lower respiratory tract infection of Candida albicans. Med Mycol 53: 153-159.

19. Bharathi M, Rani AU (2011) Pathogenic fungal isolates in sputum of HIV positive patients. J AIDS HIV Res 3: 107-113.

20. Liu ZY, Sheng RY, Li XL, Li TS, Wang AX (2003) Nosocomial fungal infections, analysis of 149 cases. Zhonghua Yi Xue Za Zhi 83: 399-402.

21. Singh T, Kashyap AK, Ahluwalia G, Chinna D, Sidhu SS (2014) Epidemiology of fungal infections in critical care setting of a tertiary care teaching hospital in North India: A prospective surveillance study. J Clin Sci Res 3: 14-25.

22. Wojciech F, Aleksandra S, Kinga A, Zygmunt A, Adam M, et al. (2015) Prevalence of yeast fungal infections in intensive care unit in Poland. Peer Pre-Prints 2: 1 